\title{
FREE VIBRATION AND BUCKLING ANALYSIS OF HIGHER ORDER LAMINATED COMPOSITE PLATES USING THE ISOGEOMETRIC APPROACH
}

\author{
Ognjen Peković, Slobodan Stupar, Aleksandar Simonović, \\ Jelena Svorcan, SrĐan Trivković \\ University of Belgrade, Faculty of Mechanical Engineering, Serbia \\ e-mail: opekovic@mas.bg.ac.rs
}

\begin{abstract}
This research paper presents a higher order isogeometric laminated composite plate finite element formulation. The isogeometric formulation is based on NURBS (non-uniform rational B-splines) basis functions of varying degree. Plate kinematics is based on the third order shear deformation theory (TSDT) of Reddy in order to avoid shear locking. Free vibration and the buckling response of laminated composite plates are obtained and efficiency of the method is considered. Numerical results with different element order are presented and the obtained results are compared to analytical and conventional numerical results as well as existing isogeometric plate finite elements.
\end{abstract}

Keywords: isogeometric, laminated composite plate, third order shear deformation theory

\section{Introduction}

Laminated composites are widely used in aerospace, marine and wind turbine industries. Recently, there has appeared a great number of general industrial products made of composites. The reasons for this are high strength-to-weight ratio, high stiffness, good dimensional stability after manufacturing and high impact, fatigue and corrosion resistance of composites. In addition to this, composite laminates possess ability to follow complex mould shapes and to be specifically tailored through optimization of ply numbers and fibre orientations through the structure so that they can meet specific needs while minimizing weight (Jones, 1999).

Laminates in general have thickness much smaller than their planar dimensions so one can use various plate theories instead of general 3D elasticity equations for their analysis (Reddy, 2004). Laminated plate theories are classified into three groups: 1) equivalent single layer theories (ESL), 2) layerwise plate theories and 3) individual-layer plate theories (Nosier et al., 1993). The equivalent single layer theories are most widely used because they provide sufficiently accurate description of the global laminate response at low computational cost. Among ESL, the classical plate theory $(\mathrm{CPT})$ is the simplest, but gives accurate results only for very thin plates since it is unable to capture transverse shear effects. The first order shear deformation theories (FSDT) give constant through thickness transverse shear strains resulting in constant transverse shear stresses through thickness, which is contradictory to the elasticity solution. In order to make up for this, one must use shear correction factors that are hard to determine. Higher order theories introduce additional unknown variables but are capable of modelling realistic transverse shear strains. Among them, the third order shear deformation theory of Reddy (1984) is the most popular among engineers. It uses quadratic variation of transverse shear strains with vanishing transverse shear stresses on the top and bottom surface, thus eliminating the need for the use of shear correction factors. 
For arbitrary shapes and boundary conditions, the governing plate equations cannot be solved analytically. Among different numerical techniques that seek approximate solutions, the finite element method became a standard tool for treatment of stress analysis problems. In FEM, the unknown field variables are approximated by linear combination of interpolation (trial or shape) functions. In the standard FEM formulation, interpolation functions are locally defined polynomials inside the element and zero everywhere outside the considered element. Most existing finite elements and all commercial codes use Lagrangian $\left(C^{0}\right.$ interelement continuity) and Hermitian ( $C^{1}$ interelement continuity) basis functions.

A great need exists in industry for integration of the manufacturing process from conceptual phase and design (by means of computer-aided design (CAD)) through analysis (by using computer aided engineering (CAE) tools) to manufacturing (done on CNC machines trough computer aided manufacturing (CAM)). CAD and CAM industries rely on the use of NURBS geometry (Piegl, 1997; Rogers, 2001) for shape representation, thus CAD/CAM integration is relatively straightforward. Although specialized CAD/CAM/CAE systems exist for the last 20-25 years (PTC Creo, CATIA V5...) communication between CAD and CAE is not straightforward, and it is necessary to build a new finite element model in order to run the necessary analysis. This task takes up to $80 \%$ of total analysis time and is therefore one of the major bottlenecks in CAD/CAE/CAM integration (Cottrell et al., 2009).

In order to overcome those difficulties, a new technique formally named isogeometric analysis is proposed (Hughes et al., 2005). It allows the execution of analysis on geometrical CAD model. Instead of Lagrange or Hermit polynomial basis functions, the isogeometric finite element method relies on NURBS basis functions, same as almost every CAD or CAM package. NURBS offers general mathematical representation of both analytical geometric objects and freeform geometry. Recently, several research papers have appeared that used the isogeometric approach for plate and shell analysis (Kiendl et al., 2009; Benson et al., 2011; Echter et al., 2013) and composite plate and shell analysis (Shojaee et al., 2012; Thai et al., 2012, 2013; Casanova and Gallego, 2013).

This paper presents free vibration and buckling analysis of TSDT composite plates in isogeometric framework. Isogeometric formulations of global stiffness, mass and geometrical stiffness matrix for quadratic, cubic and quartic elements are presented. All global matrices are formulated in full compliance with the TSDT of Reddy. Results are compared to other available data to demonstrate the accuracy of the proposed method.

\section{NURBS curves, basis functions and surfaces}

Non-uniform rational B-spline (NURBS) can represent arbitrary freeform shapes with analytical exactness that is needed in computer graphics (CG), CAD and CAM applications. After decades of technology improvement, NURBS provides users with great control over the object shape in an intuitive way with low memory consumption making them the most widespread technology for shape representation.

NURBS are generalizations of nonrational Bezier and nonrational B-splines curves and surfaces. Bezier curves are parametric polynomial curves defined as

$$
C(u)=\sum_{i=0}^{n} B_{i, n}(u) P_{i} \quad 0 \leqslant u \leqslant 1
$$

where $\left\{P_{i}\right\}$ are geometric coefficients (control points) and the $\left\{B_{i, n}(u)\right\}$ are the $n$ th-degree Bernstein polynomials (basis or blending functions) defined as

$$
B_{i, n}(u)=\frac{n !}{i !(n-i) !} u^{i}(1-u)^{n-i}
$$


In order to accurately represent conic sections, it is necessary to use rational functions instead of polynomials, so the $n$ th-degree rational Bezier curves are defined as follows

$$
C(u)=\frac{\sum_{i=0}^{n} B_{i, n}(u) w_{i} P_{i}}{\sum_{i=0}^{n} B_{i, n}(u) w_{i}} \quad 0 \leqslant u \leqslant 1
$$

where by $\left\{w_{i}\right\}$ we marked the weights that are scalar quantities.

The B-spline curve is a generalization of the Bezier curve defined as

$$
C(u)=\sum_{i=0}^{n} N_{i, p}(u) P_{i} \quad a \leqslant u \leqslant b
$$

where $\left\{P_{i}\right\}$ are control points and $\left\{N_{i, p}(u)\right\}$ are the $p$ th-degree B-spline basis functions (Fig. 1) that are defined on the nonuniform knot vector

$$
\mathbf{U}=[\underbrace{a, \ldots, a}_{p+1}, u_{p+1}, \ldots, u_{m-p-1}, \underbrace{b, \ldots, b}_{p+1}]
$$

The B-spline basis functions of the $p$ th-degree are defined recursively

$$
\begin{aligned}
& N_{i, 0}(u)= \begin{cases}1 & u_{i} \leqslant u<u_{i+1} \\
0 & \text { otherwise }\end{cases} \\
& N_{i, p}(u)=\frac{u-u_{i}}{u_{i+1}-u_{i}} N_{i, p-1}(u)+\frac{u_{i+p+1}-u}{u_{i+p+1}-u_{i+1}} N_{i+1, p-1}(u)
\end{aligned}
$$
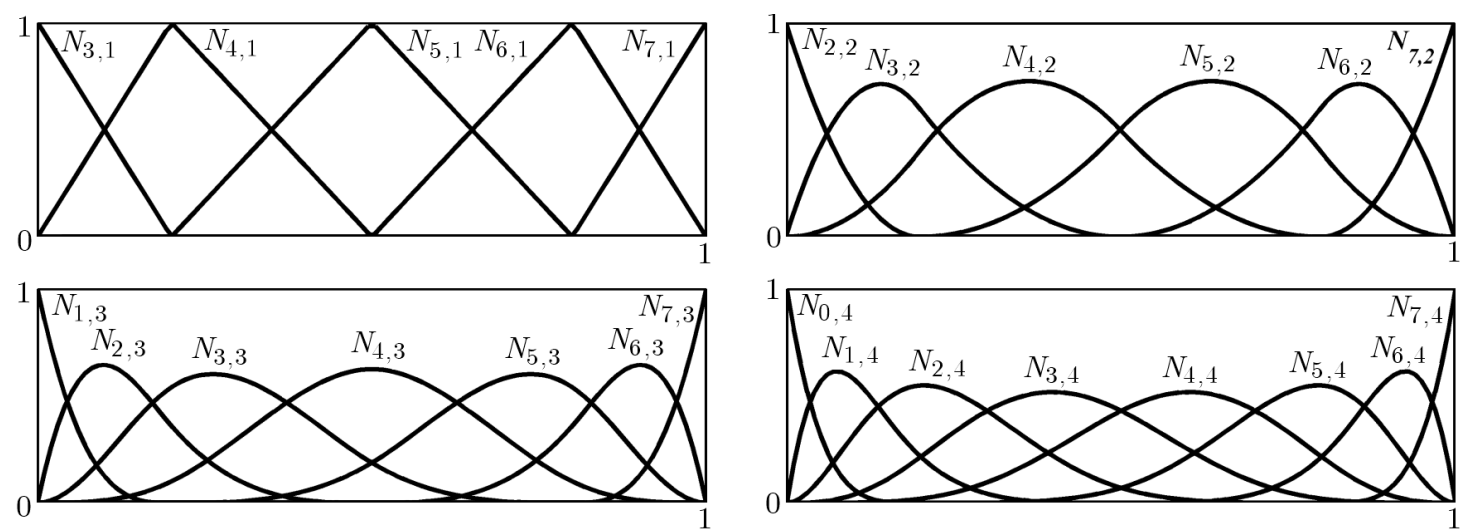

Fig. 1. Non-zero linear, quadratic, cubic and quartic B-spline basis functions defined on the open knot

$$
\text { vector } \mathbf{U}=[\underbrace{0, \ldots, 0}_{p+1}, 0.2,0.5,0.8, \underbrace{1, \ldots, 1}_{p+1}]
$$

A rational representation of the B-spline curve is called a NURBS curve. A pth-degree NURBS curve is defined analogously to (2.3) as

$$
C(u)=\frac{\sum_{i=0}^{n} N_{i, p}(u) w_{i} P_{i}}{\sum_{i=0}^{n} N_{i, p}(u) w_{i}} \quad a \leqslant u \leqslant b
$$

where $\left\{P_{i}\right\}$ are the control points, $\left\{w_{i}\right\}$ are the weights and $\left\{N_{i, p}(u)\right\}$ are the $p$ th-degree B-spline basis functions that are defined on the nonuniform knot vector given by $(2.5)$. 
If we define the rational basis functions a

$$
R_{i, p}(u)=\frac{N_{i, p}(u) w_{i}}{\sum_{j=0}^{n} N_{j, p}(u) w_{j}}
$$

we can write the NURBS curve as

$$
C(u)=\sum_{i=0}^{n} R_{i, p}(u) P_{i}
$$

It is easy to define multivariate NURBS basis functions by using the tensor product method. A NURBS surface of degree $p$ in the $u$ direction and degree $q$ in the $v$ direction is a bivariate vector-valued piecewise rational function of the form

$$
S(u, v)=\sum_{i=0}^{n} \sum_{j=0}^{m} R_{i, j}^{p, q}(u, v) P_{i, j}=\frac{\sum_{i=0}^{n} \sum_{j=0}^{m} N_{i, p}(u) N_{j, q}(v) w_{i, j} P_{i, j}}{\sum_{i=0}^{n} \sum_{j=0}^{m} N_{i, p}(u) N_{j, q}(v) w_{i, j}} \quad 0 \leqslant u, v<1
$$

where $\left\{P_{i, j}\right\}$ are the control points, $\left\{w_{i, j}\right\}$ are the weights and $\left\{R_{i, j}^{p, q}(u, v)\right\}$ are the bivariate nonrational B-spline basis function defined on the nonuniform knot vectors

$$
\mathbf{U}=[\underbrace{a, \ldots, a}_{p+1}, u_{p+1}, \ldots, u_{r-p-1}, \underbrace{b, \ldots, b}_{p+1}] \quad \mathbf{V}=[\underbrace{c, \ldots, c}_{q+1}, u_{q+1}, \ldots, u_{s-q+1}, \underbrace{d, \ldots, d}_{q+1}]
$$

where $r=n+p+1$ and $s=m+q+1$.
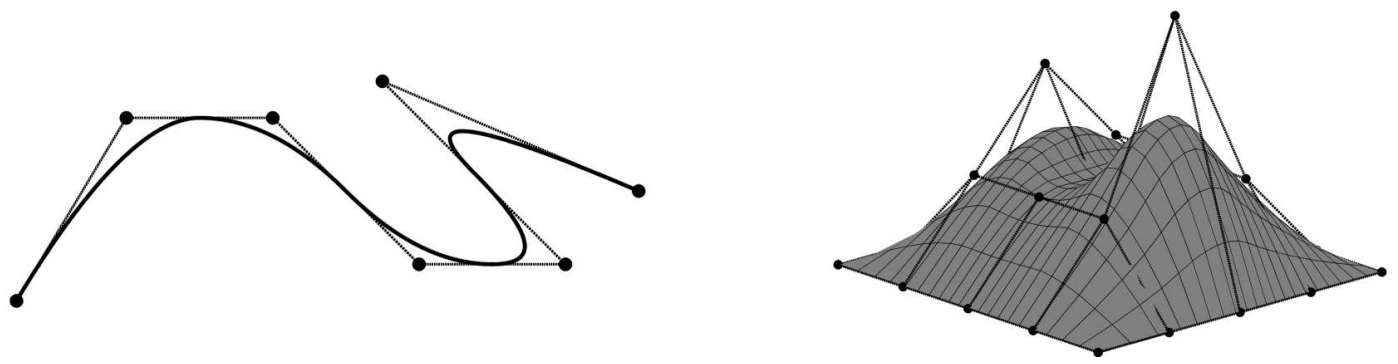

Fig. 2. Mesh of control points, the corresponding cubic NURBS curve (left) and NURBS surface (right)

\section{Equations of motion}

In the TSDT of Reddy $(1984,2004)$, the displacement field is defined as

$$
\begin{aligned}
& u(x, y, z)=u_{0}(x, y)+z \psi_{x}-\frac{4}{3 h^{2}} z^{3}\left(\psi_{x}+\frac{\partial w_{0}}{\partial x}\right) \\
& v(x, y, z)=v_{0}(x, y)+z \psi_{y}-\frac{4}{3 h^{2}} z^{3}\left(\psi_{y}+\frac{\partial w_{0}}{\partial y}\right) \\
& w(x, y, z)=w_{0}(x, y)
\end{aligned}
$$

where $u_{0}, v_{0}, w_{0}$ represent linear displacements of the midplane, $\psi_{x}, \psi_{y}$ are rotations of normals to the midplane about the $y$ and $x$-axes, respectively, and $h$ denotes the total thickness of the laminate. 
The in-plane strains $\left\{\begin{array}{lll}\varepsilon_{x x} & \varepsilon_{y y} & \gamma_{x y}\end{array}\right\}^{\mathrm{T}}$ are given as

$$
\begin{aligned}
\varepsilon_{p} & =\left\{\begin{array}{c}
\varepsilon_{x x} \\
\varepsilon_{y y} \\
\gamma_{x y}
\end{array}\right\}=\left\{\begin{array}{c}
\varepsilon_{x x}^{0} \\
\varepsilon_{y y}^{0} \\
\gamma_{x y}^{0}
\end{array}\right\}+z\left\{\begin{array}{c}
\varepsilon_{x x}^{1} \\
\varepsilon_{y y}^{1} \\
\gamma_{x y}^{1}
\end{array}\right\}+z^{3}\left\{\begin{array}{c}
\varepsilon_{x x}^{3} \\
\varepsilon_{y y}^{3} \\
\gamma_{x y}^{3}
\end{array}\right\}=\left\{\begin{array}{c}
\frac{\partial u_{0}}{\partial x} \\
\frac{\partial v_{0}}{\partial y} \\
\frac{\partial u_{0}}{\partial y}+\frac{\partial v_{0}}{\partial x}
\end{array}\right\}+z\left\{\begin{array}{c}
\frac{\partial \psi_{x}}{\partial x} \\
\frac{\partial \psi_{y}}{\partial y} \\
\frac{\partial \psi_{x}}{\partial y}+\frac{\partial \psi_{y}}{\partial x}
\end{array}\right\} \\
& -c_{1} z^{3}\left\{\begin{array}{c}
\frac{\partial \psi_{x}}{\partial x}+\frac{\partial^{2} w_{0}}{\partial x^{2}} \\
\frac{\partial \psi_{y}}{\partial y}+\frac{\partial^{2} w_{0}}{\partial y^{2}} \\
\frac{\partial \psi_{x}}{\partial y}+\frac{\partial \psi_{y}}{\partial x}+2 \frac{\partial^{2} w_{0}}{\partial x \partial y}
\end{array}\right\}
\end{aligned}
$$

and the cross plane components $\left\{\begin{array}{ll}\gamma_{y z} & \gamma_{x z}\end{array}\right\}^{\mathrm{T}}$ as

$$
\boldsymbol{\varepsilon}_{s}=\left\{\begin{array}{c}
\gamma_{y z} \\
\gamma_{x z}
\end{array}\right\}=\left\{\begin{array}{c}
\gamma_{y z}^{0} \\
\gamma_{x z}^{0}
\end{array}\right\}+z^{2}\left\{\begin{array}{c}
\gamma_{y z}^{0} \\
\gamma_{x z}^{0}
\end{array}\right\}=\left\{\begin{array}{l}
\psi_{y}+\frac{\partial w_{0}}{\partial y} \\
\psi_{x}+\frac{\partial w_{0}}{\partial x}
\end{array}\right\}-c_{2} z^{2}\left\{\begin{array}{l}
\psi_{y}+\frac{\partial w_{0}}{\partial y} \\
\psi_{x}+\frac{\partial w_{0}}{\partial x}
\end{array}\right\}
$$

with $c_{1}=4 /\left(3 h^{2}\right)$ and $c_{2}=4 /\left(h^{2}\right)$.

Constitutive relations between stresses and strains in the $k$-th lamina in the case of plane stress state, in the local coordinate system of the principle material coordinates $\left(x_{1}, x_{2}, x_{3}\right)$, where $x_{1}$ is fibre direction, $x_{2}$ in-plane normal to fibre and $x_{3}$ normal to the lamina plane, are given by

$$
\left\{\begin{array}{c}
\sigma_{1}^{(k)} \\
\sigma_{2}^{(k)} \\
\tau_{12}^{(k)} \\
\tau_{23}^{(k)} \\
\tau_{13}^{(k)}
\end{array}\right\}=\left[\begin{array}{ccccc}
Q_{11} & Q_{12} & 0 & 0 & 0 \\
Q_{12} & Q_{22} & 0 & 0 & 0 \\
0 & 0 & Q_{66} & 0 & 0 \\
0 & 0 & 0 & Q_{44} & Q_{45} \\
0 & 0 & 0 & Q_{45} & Q_{55}
\end{array}\right]^{(k)}\left\{\begin{array}{c}
\varepsilon_{1}^{(k)} \\
\varepsilon_{2}^{(k)} \\
\gamma_{12}^{(k)} \\
\gamma_{23}^{(k)} \\
\gamma_{13}^{(k)}
\end{array}\right\}
$$

The quantities $Q_{i j}^{(k)}$ are called the plane reduced stiffness components and are given in terms of material properties of each layer as

$$
\begin{aligned}
& Q_{11}^{(k)}=\frac{E_{1}^{(k)}}{1-\nu_{12}^{(k)} \nu_{21}^{(k)}} \quad Q_{12}^{(k)}=\frac{\nu_{12}^{(k)} E_{2}^{(k)}}{1-\nu_{12}^{(k)} \nu_{21}^{(k)}} \quad Q_{22}^{(k)}=\frac{E_{2}^{(k)}}{1-\nu_{12}^{(k)} \nu_{21}^{(k)}} \\
& Q_{66}^{(k)}=G_{12}^{(k)} \quad Q_{44}^{(k)}=G_{23}^{(k)} Q_{55}^{(k)}=G_{13}^{(k)}
\end{aligned}
$$

$E_{1}^{(k)}, E_{2}^{(k)}$ are Young's moduli, $\nu_{12}^{(k)}, \nu_{21}^{(k)}$ are Poisson's coefficients and $G_{12}^{(k)}, G_{23}^{(k)}, G_{13}^{(k)}$ are shear moduli of the lamina.

Composite laminates are usually made of several orthotropic layers (laminae) of different orientation. In order to express constitutive relations in the referent laminate $(x, y, z)$ coordinate system (Fig. 3), the lamina constitutive relations are transformed as

$$
\left\{\begin{array}{c}
\sigma_{x x}^{(k)} \\
\sigma_{y y}^{(k)} \\
\tau_{x y}^{(k)} \\
\tau_{y z}^{(k)} \\
\tau_{x z}^{(k)}
\end{array}\right\}=\left[\begin{array}{ccccc}
\bar{Q}_{11} & \bar{Q}_{12} & \bar{Q}_{16} & 0 & 0 \\
\bar{Q}_{12} & \bar{Q}_{22} & \bar{Q}_{26} & 0 & 0 \\
\bar{Q}_{16} & \bar{Q}_{26} & \bar{Q}_{66} & 0 & 0 \\
0 & 0 & 0 & \bar{Q}_{44} & \bar{Q}_{45} \\
0 & 0 & 0 & \bar{Q}_{45} & \bar{Q}_{55}
\end{array}\right]^{(k)}\left\{\begin{array}{c}
\varepsilon_{x x}^{(k)} \\
\varepsilon_{y y}^{(k)} \\
\gamma_{x y}^{(k)} \\
\gamma_{y z}^{(k)} \\
\gamma_{x z}^{(k)}
\end{array}\right\}
$$


where $\bar{Q}_{i j}$ are the lamina plane stress reduced stiffness components in the laminate coordinate system defined as (Reddy, 2004)

$$
\begin{aligned}
& \left\{\begin{array}{l}
{\overline{\bar{Q}_{11}}}_{\bar{Q}_{22}}{ }^{(k)} \bar{Q}_{16} \\
\bar{Q}_{26} \\
\bar{Q}_{66}
\end{array}\right\}=\left[\begin{array}{cccc}
m^{4} & n^{4} & 2 m^{2} n^{2} & 4 m^{2} n^{2} \\
n^{4} & m^{4} & 2 m^{2} n^{2} Q_{12} & 4 m^{2} n^{2} \\
m^{3} & -m n^{3} & m n^{3}-m^{3} n & 2\left(m n^{3}-m^{3} n\right) \\
m n^{3} & -m^{3} n & m^{3} n-m n^{3} & 2\left(m^{3} n-m n^{3}\right) \\
m^{2} n^{2} & m^{2} n^{2} & -2 m^{2} n^{2} & \left(m^{2}-n^{2}\right)^{2}
\end{array}\right]^{(k)}\left\{\begin{array}{l}
Q_{11} \\
Q_{22} \\
Q_{12} \\
Q_{66}
\end{array}\right\} \\
& \left\{\begin{array}{cc}
\bar{Q}_{44} \\
\bar{Q}_{45} \\
\bar{Q}_{55}
\end{array}\right\}=\left[\begin{array}{cc}
m^{2} & n^{2} \\
-m n & m n \\
n^{2} & m^{2}
\end{array}\right]^{(k)}\left\{\begin{array}{l}
Q_{44} \\
Q_{55}
\end{array}\right\}^{(k)}
\end{aligned}
$$

with $m$ and $n$ denote cosine and sine of the angle $\theta$ between the global axis $x$ and local axis $x_{1}$ (Fig. 3).

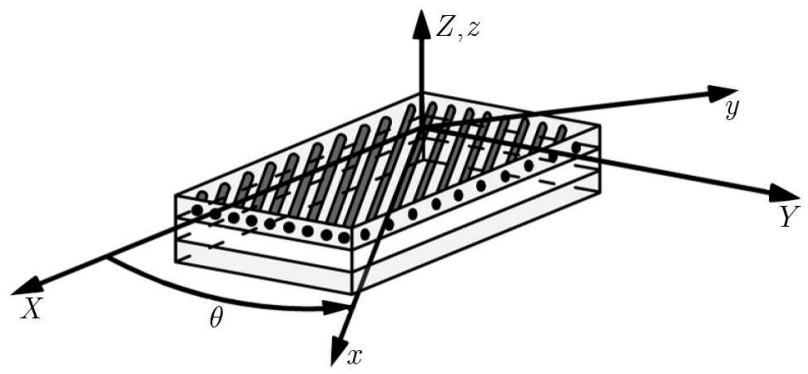

Fig. 3. Local and global coordinate systems of a laminate

The dynamic form of the principle of virtual work in matrix form is given by

$$
\int_{\Omega} \delta \varepsilon_{p}^{\mathrm{T}} \mathcal{D} \varepsilon_{p} d \Omega+\int_{\Omega} \delta \varepsilon_{s}^{\mathrm{T}} \mathcal{D}^{s} \varepsilon_{s} d \Omega=\int_{\Omega} \delta \mathbf{u}^{\mathrm{T}} \mathbf{m} \ddot{\mathbf{u}} d \Omega
$$

where $\mathbf{m}$ is defined as

$$
\mathbf{m}=\left[\begin{array}{ccccccc}
I_{0} & 0 & 0 & J_{1} & 0 & -c_{1} I_{3} & 0 \\
0 & I_{0} & 0 & 0 & J_{1} & 0 & -c_{1} I_{3} \\
0 & 0 & I_{0} & 0 & 0 & 0 & 0 \\
J_{1} & 0 & 0 & K_{2} & 0 & -c_{1} I_{4} & 0 \\
0 & J_{1} & 0 & 0 & K_{2} & 0 & -c_{1} I_{4} \\
-c_{1} I_{3} & 0 & 0 & -c_{1} I_{4} & 0 & c_{1}^{2} I_{6} & 0 \\
0 & -c_{1} I_{3} & 0 & 0 & -c_{1} I_{4} & 0 & c_{1}^{2} I_{6}
\end{array}\right]
$$

with

$$
\begin{aligned}
& \left(I_{0}, I_{1}, I_{2}, I_{3}, I_{4}, I_{6}\right)=\sum_{k=1}^{N} \int_{-h / 2}^{h / 2} \rho^{(k)}\left(1, z, z^{2}, z^{3}, z^{4}, z^{6}\right) d z \\
& J_{1}=I_{1}-c_{1} I_{3} \quad K_{2}=I_{2}-2 c_{1} I_{4}+c_{1}^{2} I_{6}
\end{aligned}
$$

Matrices that relate the stress resultants to the strains are given as

$$
\mathcal{D}=\left[\begin{array}{lll}
\mathbf{A} & \mathbf{B} & \mathbf{E} \\
\mathbf{B} & \mathbf{D} & \mathbf{F} \\
\mathbf{E} & \mathbf{F} & \mathbf{H}
\end{array}\right] \quad \mathcal{D}^{s}=\left[\begin{array}{ll}
\mathbf{A}^{s} & \mathbf{D}^{s} \\
\mathbf{D}^{s} & \mathbf{F}^{s}
\end{array}\right]
$$


with

$$
\begin{aligned}
& \left(A_{i j}, B_{i j}, D_{i j}, E_{i j}, F_{i j}, H_{i j}\right)=\sum_{k=1}^{N} \int_{-h / 2}^{h / 2} \bar{Q}_{i j}^{(k)}\left(1, z, z^{2}, z^{3}, z^{4}, z^{6}\right) d z \\
& \left(A_{i j}^{s}, D_{i j}^{s}, F_{i j}^{s}\right)=\sum_{k=1}^{N} \int_{-h / 2}^{h / 2} \bar{Q}_{i j}^{(k)}\left(1, z^{2}, z^{4}\right) d z \\
& \mathbf{u}^{\mathrm{T}}=\left\{\begin{array}{lllllll}
u_{0} & v_{0} & w_{0} & \psi_{x} & \psi_{y} & \frac{\partial w_{0}}{\partial x} & \frac{\partial w_{0}}{\partial y}
\end{array}\right\}^{\mathrm{T}}
\end{aligned}
$$

For buckling analysis, the weak form of the virtual work principle in the matrix form is stated as

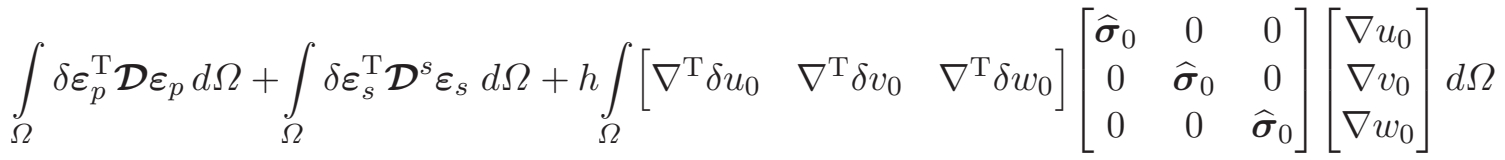

$$
\begin{aligned}
& +\frac{h^{3}}{12} \int_{\Omega}\left[\begin{array}{ll}
\nabla^{\mathrm{T}} \delta \psi_{x} & \nabla^{\mathrm{T}} \delta \psi_{y}
\end{array}\right]\left[\begin{array}{cc}
\widehat{\boldsymbol{\sigma}}_{0} & 0 \\
0 & \widehat{\boldsymbol{\sigma}}_{0}
\end{array}\right]\left[\begin{array}{c}
\nabla \psi_{x} \\
\nabla \psi_{y}
\end{array}\right] d \Omega=0
\end{aligned}
$$

where $\nabla^{\mathrm{T}}=\left[\begin{array}{ll}\partial / \partial x & \partial / \partial y\end{array}\right]$ is the gradient operator and $\widehat{\boldsymbol{\sigma}}_{0}=\left[\begin{array}{cc}\sigma_{x}^{0} & \tau_{x y}^{0} \\ \tau_{x y}^{0} & \sigma_{y}^{0}\end{array}\right]$ is the matrix of in-plane pre-buckling stresses.

\section{Isogeometric finite element formulation of TSDT laminated plate}

In TSDT, the field variables are inplane displacements, transverse displacements and rotations at control points. By using isogeometric paradigm, the same NURBS basis functions that are used to describe plate geometry are used for the interpolation of field variables

$$
\mathbf{u}=\left\{\begin{array}{l}
u_{0} \\
v_{0} \\
w_{0} \\
\psi_{x} \\
\psi_{y}
\end{array}\right\}=\sum_{I=1}^{n \times m}\left[\begin{array}{ccccc}
N_{I} & 0 & 0 & 0 & 0 \\
0 & N_{I} & 0 & 0 & 0 \\
0 & 0 & N_{I} & 0 & 0 \\
0 & 0 & 0 & N_{I} & 0 \\
0 & 0 & 0 & 0 & N_{I}
\end{array}\right]\left\{\begin{array}{c}
u_{0 I} \\
v_{0 I} \\
w_{0 I} \\
\psi_{x I} \\
\psi_{y I}
\end{array}\right\}=\sum_{I=1}^{n \times m} N_{I} q_{I}
$$

where $n \times m$ is the number of control points (basis functions), $N_{I}$ are rational basis functions and $q_{I}$ are degrees of freedom associated with the control point $I$.

The in-plane strains and shear strains are obtained using Eqs. (3.2),(3.3) and (4.1) as

$$
\varepsilon_{p}=\sum_{I}\left[\mathbf{B}_{I}^{0}+z \mathbf{B}_{I}^{1}-c_{1} z^{3} \mathbf{B}_{I}^{3}\right] \mathbf{q}_{I} \quad \varepsilon_{s}=\sum_{I}\left[\mathbf{B}_{I}^{S 0}-c_{2} z^{2} \mathbf{B}_{I}^{S 2}\right] \mathbf{q}_{I}
$$

where

$$
\begin{aligned}
\mathbf{B}^{0} & =\left[\begin{array}{ccccc}
N, x & 0 & 0 & 0 & 0 \\
0 & N, y & 0 & 0 & 0 \\
N, y & N, x & 0 & 0 & 0
\end{array}\right] \quad \mathbf{B}^{1}=\left[\begin{array}{ccccc}
0 & 0 & 0 & N, x & 0 \\
0 & 0 & 0 & 0 & N, y \\
0 & 0 & 0 & N, y & N, x
\end{array}\right] \\
\mathbf{B}^{3} & =\left[\begin{array}{ccccc}
0 & 0 & N, x x & N, x & 0 \\
0 & 0 & N, y y & 0 & N, y \\
0 & 0 & 2 N, x y & N, y & N, x
\end{array}\right]
\end{aligned}
$$


and

$$
\mathbf{B}^{S 0}=\mathbf{B}^{S 2}=\left[\begin{array}{llllc}
0 & 0 & N, y & 0 & N \\
0 & 0 & N, x & N & 0
\end{array}\right]
$$

where $N, x$ and $N, y$ denote the first and $N, x x, N, y y, N, x y$ second derivatives of $N$ with respect to $x$ and $y$.

For free vibration analysis, we can write

$$
\left(\mathbf{K}-\omega^{2} \mathbf{M}\right) \mathbf{q}=\mathbf{0}
$$

and for buckling analysis, we get

$$
\left(\mathbf{K}-\lambda_{c r} \mathbf{K}_{g}\right) \mathbf{q}=\mathbf{0}
$$

where $\mathbf{K}$ is the global stiffness matrix defined as

$$
\mathbf{K}=\int_{\Omega}\left[\begin{array}{l}
\mathbf{B}^{0} \\
\mathbf{B}^{1} \\
\mathbf{B}^{3}
\end{array}\right]^{\mathrm{T}}\left[\begin{array}{lll}
\mathbf{A} & \mathbf{B} & \mathbf{E} \\
\mathbf{B} & \mathbf{D} & \mathbf{F} \\
\mathbf{E} & \mathbf{F} & \mathbf{H}
\end{array}\right]\left[\begin{array}{l}
\mathbf{B}^{0} \\
\mathbf{B}^{1} \\
\mathbf{B}^{3}
\end{array}\right]+\left[\begin{array}{l}
\mathbf{B}^{s 0} \\
\mathbf{B}^{s 2}
\end{array}\right]^{\mathrm{T}}\left[\begin{array}{ll}
\mathbf{A}^{s} & \mathbf{D}^{s} \\
\mathbf{D}^{s} & \mathbf{F}^{s}
\end{array}\right]\left[\begin{array}{l}
\mathbf{B}^{s 0} \\
\mathbf{B}^{s 2}
\end{array}\right] d \Omega
$$

The global mass matrix $\mathbf{M}$ is given by

$$
\mathbf{M}=\int_{\Omega} \mathbf{N}_{m}^{\mathrm{T}} \mathbf{m} \mathbf{N}_{m} d \Omega
$$

with

$$
\mathbf{N}_{m}=\left[\begin{array}{ccccccc}
N_{I} & 0 & 0 & 0 & 0 & 0 & 0 \\
0 & N_{I} & 0 & 0 & 0 & 0 & 0 \\
0 & 0 & N_{I} & 0 & 0 & N_{I}, x & N_{I}, y \\
0 & 0 & 0 & N_{I} & 0 & 0 & 0 \\
0 & 0 & 0 & 0 & N_{I} & 0 & 0
\end{array}\right]^{\mathrm{T}}
$$

The global geometrical stiffness matrix $\mathbf{K}_{g}$ that takes into account the contribution of shear strains is given by

$$
\mathbf{K}_{g}=\int_{\Omega} \mathbf{N}_{g}^{\mathrm{T}} \mathbf{I}_{g} \mathbf{N}_{g} d \Omega
$$

with

$$
\mathbf{N}_{g}=\left[\begin{array}{ccccc}
\nabla N & 0 & 0 & 0 & 0 \\
0 & \nabla N & 0 & 0 & 0 \\
0 & 0 & \nabla N & 0 & 0 \\
0 & 0 & 0 & \nabla N & 0 \\
0 & 0 & 0 & 0 & \nabla N \\
0 & 0 & N_{I}, x x & N_{I}, x & 0 \\
0 & 0 & N_{I}, x y & N_{I}, y & 0 \\
0 & 0 & N_{I}, x y & 0 & N_{I}, x \\
0 & 0 & N_{I}, y y & 0 & N_{I}, y
\end{array}\right]
$$$$
\mathbf{I}_{g}=\left[\begin{array}{ccccccc}
\mathbf{I}_{g 0} & 0 & 0 & 0 & 0 & 0 & 0 \\
0 & \mathbf{I}_{g 0} & 0 & 0 & 0 & 0 & 0 \\
0 & 0 & \mathbf{I}_{g 0} & 0 & 0 & 0 & 0 \\
0 & 0 & 0 & \mathbf{I}_{g 2} & 0 & \mathbf{I}_{g 4} & 0 \\
0 & 0 & 0 & 0 & \mathbf{I}_{g 2} & 0 & \mathbf{I}_{g 4} \\
0 & 0 & 0 & \mathbf{I}_{g 4} & 0 & \mathbf{I}_{g 6} & 0 \\
0 & 0 & 0 & 0 & \mathbf{I}_{g 4} & 0 & \mathbf{I}_{g 6}
\end{array}\right]
$$

and

$$
\begin{aligned}
& \nabla N=\left[\begin{array}{ll}
N_{I}, x & N_{I}, y
\end{array}\right]^{\mathrm{T}} \quad \mathbf{I}_{g 0}=h \widehat{\boldsymbol{\sigma}}_{0} \quad \mathbf{I}_{g 2}=\frac{h^{3}}{12} \widehat{\boldsymbol{\sigma}}_{0} \\
& \mathbf{I}_{g 4}=\frac{h^{5}}{80}\left[\begin{array}{cc}
\sigma_{x}^{0} & -\tau_{x y}^{0} \\
-\tau_{x y}^{0} & \sigma_{y}^{0}
\end{array}\right] \quad \mathbf{I}_{g 6}=\frac{h^{7}}{448}\left[\begin{array}{cc}
\sigma_{x}^{0} & -\tau_{x y}^{0} \\
-\tau_{x y}^{0} & \sigma_{y}^{0}
\end{array}\right]
\end{aligned}
$$




\section{Free vibration analysis of laminated composite plates}

In this Section, the performance of quadratic, cubic and quartic TSDT isogeometric elements in the free vibration analysis is studied. Standard benchmark problems with various plate shapes, boundary conditions, material characteristics and thickness are solved using the proposed method, and the results are compared to other available ones.

\subsection{Square composite plates}

We consider [0/90], [0/90/0] and [0/90/0/90] cross-ply laminates. Each ply is made of an orthotropic material with the following characteristics: $E_{1}=1.73 \cdot 10^{5} \mathrm{MPa}, E_{2}=3.31 \cdot 10^{4} \mathrm{MPa}$, $G_{12}=9.38 \cdot 10^{3} \mathrm{MPa}, G_{13}=8.27 \cdot 10^{3} \mathrm{MPa}, G_{23}=3.24 \cdot 10^{3} \mathrm{MPa}$ and $\nu_{12}=0.036$. Mass density $\rho$ is equal to one. The plate is simply supported on all edges, and all layers are assumed to be of the same thickness and density. The plates length-to-width ratio is $a / b=1$ and the width-to-thickness ratio is $b / h=10$. The normalized frequency is defined as $\bar{\omega}=(\omega h) \sqrt{\rho / E_{2}}$.

In Tables 1, 2 and 3, we present four dimensionless natural frequencies that correspond to the values of Fourier integers $m, n=1,2$ for [0/90], [0/90/0] and [0/90/0/90] laminates, respectively. We compare the results of quadratic, cubic and quartic IGA TSDT elements with the exact 3D elasticity solution and the analytical solutions of TSDT, FSDT and CPT theories given by Nosier et al. (1993). The shear correction factor for FSDT theory is taken to be $\pi^{2} / 12$. In this example, the plate is modeled with $8 \times 8$ elements.

The results obtained with quadratic elements are in very good agreement with the analytical solution based on TSDT theory of Reddy. We see that the results obtained with cubic and quartic elements are quite similar and differ slightly from the quadratic elements solutions.

Table 1. Non-dimensional frequency parameter $\bar{\omega}$ of the $[0 / 90]$ laminate

\begin{tabular}{|l|c|c|c|c|c|c|c|}
\hline & Exact & TSDT & FSDT & CPT & $\begin{array}{c}\text { IGA } \\
\text { TSDT } \\
\text { quadratic }\end{array}$ & $\begin{array}{c}\text { IGA } \\
\text { TSDT } \\
\text { cubic }\end{array}$ & $\begin{array}{c}\text { IGA } \\
\text { TSDT } \\
\text { quartic }\end{array}$ \\
\hline \hline$m, n=1$ & 0.06027 & 0.06057 & 0.6038 & 0.06513 & 0.06056 & 0.06053 & 0.06053 \\
\hline $\begin{array}{l}m, n=1,2 \\
m, n=2,1\end{array}$ & 0.14539 & 0.14681 & 0.14545 & 0.17744 & 0.14703 & 0.14664 & 0.14663 \\
\hline$m, n=2$ & 0.20229 & 0.20482 & 0.20271 & 0.25814 & 0.20508 & 0.20449 & 0.20448 \\
\hline
\end{tabular}

Table 2. Non-dimensional frequency parameter $\bar{\omega}$ of the $[0 / 90 / 0]$ laminate

\begin{tabular}{|l|c|c|c|c|c|c|c|}
\hline & Exact & TSDT & FSDT & CPT & $\begin{array}{c}\text { IGA } \\
\text { TSDT } \\
\text { quadratic }\end{array}$ & $\begin{array}{c}\text { IGA } \\
\text { TSDT } \\
\text { cubic }\end{array}$ & $\begin{array}{c}\text { IGA } \\
\text { TSDT } \\
\text { quartic }\end{array}$ \\
\hline \hline$m, n=1$ & 0.06715 & 0.06839 & 0.06931 & 0.07769 & 0.06837 & 0.06835 & 0.06835 \\
\hline$m, n=1,2$ & 0.12811 & 0.13010 & 0.12886 & 0.15185 & 0.13014 & 0.12993 & 0.12993 \\
\hline$m, n=2,1$ & 0.17217 & 0.17921 & 0.18674 & 0.26599 & 0.17957 & 0.17908 & 0.17907 \\
\hline$m, n=2$ & 0.20798 & 0.21526 & 0.22055 & 0.31077 & 0.21551 & 0.21494 & 0.21494 \\
\hline
\end{tabular}

\subsection{Circular composite plates}

Next, we consider a symmetric four-layer laminated circular plate with $\left[\theta^{\circ} /-\theta^{\circ} /-\theta^{\circ} / \theta^{\circ}\right]$ stacking sequence and clamped boundaries.

The material of each ply has the following characteristics: $E_{1}=40 E_{2}, G_{12}=G_{13}=0.6 E_{2}$, $G_{23}=0.5 E_{2}, \nu_{12}=0.25, \rho=1$. Fiber orientation angles are $\theta=0^{\circ}, 15^{\circ}, 30^{\circ}, 45^{\circ}$ and the 
Table 3. Non-dimensional frequency parameter $\bar{\omega}$ of the $[0 / 90 / 0 / 90]$ laminate

\begin{tabular}{|l|c|c|c|c|c|c|c|}
\hline & Exact & TSDT & FSDT & CPT & $\begin{array}{c}\text { IGA } \\
\text { TSDT } \\
\text { quadratic }\end{array}$ & $\begin{array}{c}\text { IGA } \\
\text { TSDT } \\
\text { cubic }\end{array}$ & $\begin{array}{c}\text { IGA } \\
\text { TSDT } \\
\text { quartic }\end{array}$ \\
\hline \hline$m, n=1$ & 0.06621 & 0.06789 & 0.06791 & 0.07474 & 0.06787 & 0.06785 & 0.06785 \\
\hline $\begin{array}{l}m, n=1,2 \\
m, n=2,1\end{array}$ & 0.15194 & 0.16065 & 0.16066 & 0.20737 & 0.16085 & 0.16048 & 0.16048 \\
\hline$m, n=2$ & 0.20841 & 0.22108 & 0.22097 & 0.29824 & 0.22133 & 0.22077 & 0.22076 \\
\hline
\end{tabular}

diameter-to-thickness ratio is 10 . In order to represent the circular plate geometry, we used quadratic basis functions with knot vectors $\mathbf{U}=[0,0,0,1,1,1]$ and $\mathbf{V}=[0,0,0,1,1,1]$. We chose an appropriate control polygon in order to get a desirable distribution of the parametric curves. The control polygon and resulting mesh are shown in Fig. 4. The results for a $8 \times 8$ element mesh are presented in Table 4 and compared with the results obtained with MISQ20 elements (Nguyen-Van et al., 2008), MLSDQ elements (Liew et al., 2003) and the IGA FSDT results obtained by Thai et al. (2012). There is a good agreement between the results. The first six mode shapes of the quartic $\left[45^{\circ} /-45^{\circ} /-45^{\circ} / 45^{\circ}\right]$ clamped laminated circular plate are shown in Fig. 5.
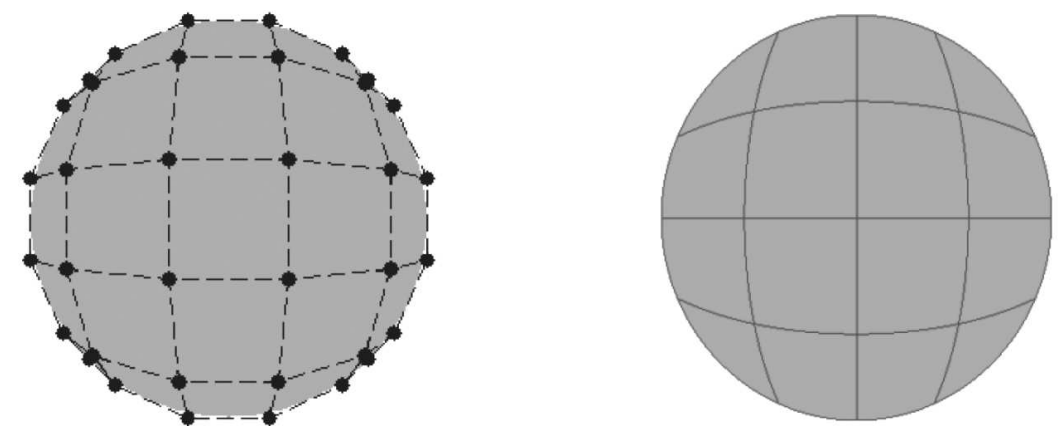

Fig. 4. The control polygon and knot plot of a quadratic circular plate with 4 non-zero knot spans
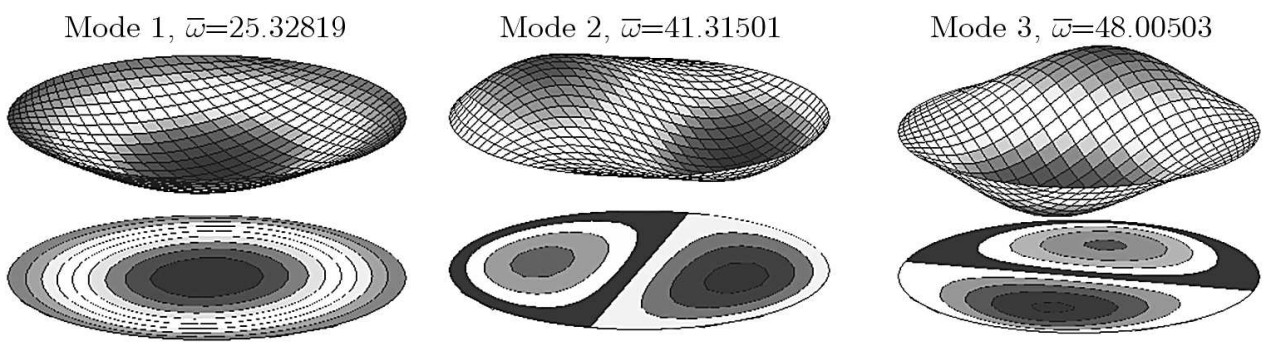

Mode $4, \bar{\omega}=59.65923$

Mode $5, \bar{\omega}=65.27138$
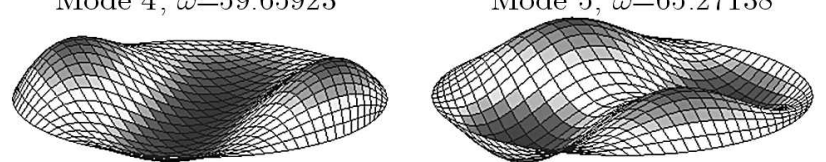

Mode $6, \bar{\omega}=73.0149$
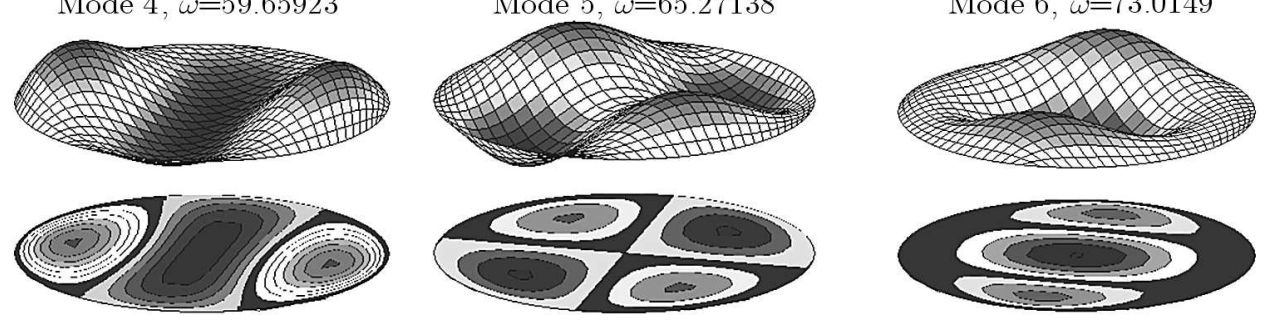

Fig. 5. First six mode shapes of a quartic $\left[45^{\circ} /-45^{\circ} /-45^{\circ} / 45^{\circ}\right]$ clamped laminated circular plate 
Table 4. Non-dimensional frequency parameter $\bar{\omega}$ of the $\left[\theta^{\circ} /-\theta^{\circ} /-\theta^{\circ} / \theta^{\circ}\right]$ circular laminated plate

\begin{tabular}{|c|c|c|c|c|c|c|c|}
\hline \multirow{2}{*}{$\theta^{\circ}$} & \multirow{2}{*}{ Method } & \multicolumn{6}{|c|}{ Modes } \\
\hline & & 1 & 2 & 3 & 4 & 5 & 6 \\
\hline \multirow[t]{8}{*}{$\overline{00^{\circ}}$} & SDT - MISQ20 & 22.123 & 29.768 & 41.726 & 42.805 & 50.756 & 256.950 \\
\hline & SDT - MLSDQ & 2.211 & 9.651 & 41.101 & 42.635 & 50.309 & 54.553 \\
\hline & $\overline{\text { GA FSDT quadrs }}$ & 2.0989 & 29.5409 & 40.8126 & 42.5447 & 50.2975 & 54.7732 \\
\hline & IGA FSDT cubic & 22.1110 & 29.5550 & 40.8150 & 42.5650 & 50.3201 & 54.7332 \\
\hline & IGA FSDT quartic & 22.1227 & 29.5735 & 40.8410 & 42.5854 & 50.3478 & 54.7609 \\
\hline & IGA TSDT q & 22.8351 & 31.4480 & 45.5659 & 48.1996 & 49.5516 & 56.7189 \\
\hline & IGA TSDT cubic & 22.6745 & 31.2413 & 45.3267 & 48.1985 & 49.4442 & 56.5244 \\
\hline & $\overline{\mathrm{GA}} \mathrm{TSDT}$ & 22.6127 & 31.0741 & 45.0771 & 48.1983 & 49.4310 & 56.4541 \\
\hline \multirow[t]{8}{*}{$15^{\circ}$} & FSDT - MISQ20 & 22.698 & 31.568 & 43.635 & 44.318 & 53.468 & 60.012 \\
\hline & FSDT - MLSDQ & 22.774 & 31.455 & 43.350 & 43.469 & 52.872 & 57.386 \\
\hline & GA FSDT quadr & 2.6500 & 1.3012 & 43.3124 & 43.3833 & 52.8952 & 57.8347 \\
\hline & IGA F & 22.6626 & 31.3166 & 43.3335 & 43.3899 & 52.9197 & 57.8064 \\
\hline & IGA FSDT quartic & 22.6751 & 31.3359 & 43.3550 & 43.4165 & 52.9486 & 57.8349 \\
\hline & IGA TSDT quadrat & 23.4537 & 33.6251 & 48.4304 & 49.4626 & 58.8442 & 66.4838 \\
\hline & GA TSDT cubic & 23.2857 & 33.4227 & 48.1945 & 49.3160 & 58.5463 & 66.1343 \\
\hline & IGA TSDT & 23.2140 & 33.2644 & 47.9875 & 49.3001 & 58.4857 & 65.9376 \\
\hline \multirow[t]{8}{*}{$30^{\circ}$} & FSDT - MIs & 24.046 & 36.399 & 44.189 & 52.028 & 57.478 & 67.099 \\
\hline & FSDT - MLSDQ & 24.071 & 36.153 & 43.968 & 51.074 & 56.315 & 66.220 \\
\hline & IGA FSDT quadra & 3.9428 & 35.9896 & 43.7948 & 50.9574 & 56.6770 & 66.0745 \\
\hline & IGA FSDT cubic & 3.9565 & 36.0085 & 43.8164 & 50.9745 & 56.7038 & 66.1011 \\
\hline & IGA FSDT quartic & 23.9703 & 36.0298 & 43.8390 & 51.0024 & 56.7337 & 66.1316 \\
\hline & IGA TSDT quadrat & 24.9036 & 38.7086 & 48.9210 & 56.0703 & 62.7850 & 75.2087 \\
\hline & IGA TSDT cubic & 24.7076 & 38.5058 & 48.7678 & 55.8127 & 62.4374 & 74.7126 \\
\hline & IGA I & 24.6221 & 38.4000 & 48.7367 & 55.7171 & 62.3863 & 74.6206 \\
\hline \multirow[t]{8}{*}{$45^{\circ}$} & FSDT - MISQ20 & 24.766 & 39.441 & 43.817 & 57.907 & 57.945 & 66.297 \\
\hline & FSDT - MLSDQ & 24.752 & 39.181 & 43.607 & 56.759 & 56.967 & 65.571 \\
\hline & IGA FSDT quadratic & 24.6335 & 38.9379 & 43.4120 & 56.8708 & 56.9251 & 65.2751 \\
\hline & IGA FSDT C & 24.6478 & 38.9591 & 43.4330 & 56.8937 & 56.9531 & 65.3002 \\
\hline & IGA FSDT quartic & 24.6622 & 38.9814 & 43.4559 & 56.9205 & 56.9844 & 65.3320 \\
\hline & IGA TSDT quadrat & 25.6205 & 41.4886 & 48.2065 & 59.9176 & 65.6484 & 73.5627 \\
\hline & IGA TSDT cubic & 25.4140 & 41.3547 & 48.0552 & 59.6995 & 65.2816 & 73.0792 \\
\hline & IGA TSDT quartic & 25.3282 & 41.3150 & 48.0050 & 59.6592 & 65.2714 & 73.0149 \\
\hline
\end{tabular}

\section{Buckling analysis of composite plate}

\subsection{Square plate under uniaxial compression}

We consider a symmetric four-layer $\left[0^{\circ} / 90^{\circ} / 0^{\circ} / 90^{\circ}\right]$ cross-ply plate with simply supported (SS-1) boundary conditions on all sides (Fig. 6). The plate material is the same as in the previous example. In Table 5, we present the convergence study of a dimensionless buckling load factor defined as $\bar{\lambda}=\lambda_{c r} a^{2} /\left(E_{2} h^{3}\right)$ with the edge-to-thickness ratio equal to 10, where $a$ is edge length, $h$ is total thickness of the laminate, $\lambda_{c r}$ is the critical load factor and $E_{2}$ is the elastic modulus. In Table 6 , the results for different edge-to-thickness ratios and an $8 \times 8$ element mesh are compared with the analytical solutions based on CPT, FSDT and TSDT theories given by Reddy (2004) and with IGA FSDT solutions by Thai et al. (2012). The obtained results agree remarkably with the other available ones. 

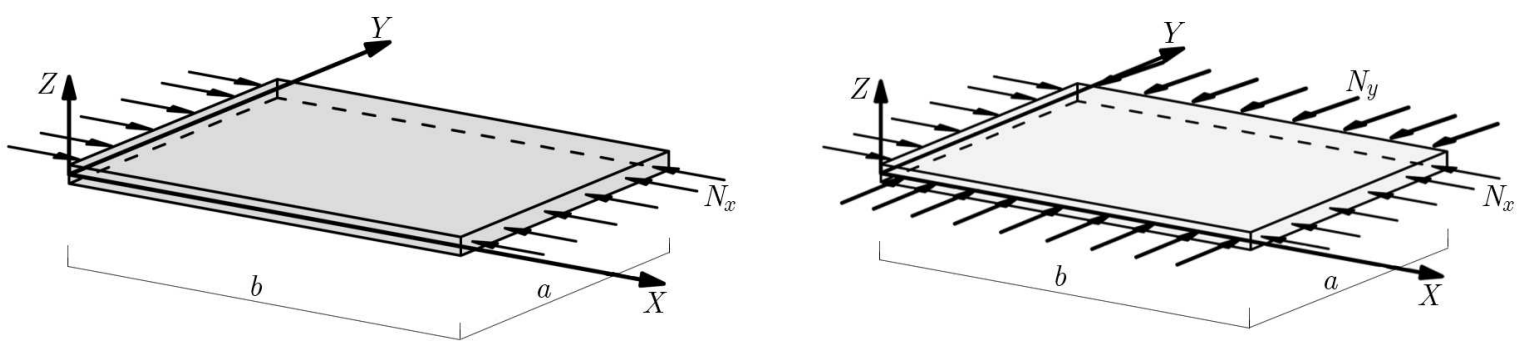

Fig. 6. Simply supported laminated plate under uniaxial (left) and biaxial (right) compression

Table 5. Normalized critical buckling load of the simply supported cross-ply $\left[0^{\circ} / 90^{\circ} / 90^{\circ} / 0^{\circ}\right]$

\begin{tabular}{|l|c|c|c|c|}
\hline \multirow{2}{*}{ Method } & \multicolumn{4}{|c|}{ Mesh } \\
\cline { 2 - 5 } & $4 \times 4$ & $8 \times 8$ & $16 \times 16$ & $32 \times 32$ \\
\hline \hline IGA TSDT quadratic & 23.2336 & 23.1725 & 23.1596 & 23.1563 \\
\hline IGA TSDT cubic & 23.1563 & 23.1551 & 23.1551 & 23.1551 \\
\hline IGA TSDT quartic & 23.1551 & 23.1551 & 23.1551 & 23.1551 \\
\hline
\end{tabular}

Table 6. Normalized critical buckling load of the simply supported cross-ply $\left[0^{\circ} / 90^{\circ} / 90^{\circ} / 0^{\circ}\right]$ plate

\begin{tabular}{|l|c|c|c|c|c|}
\hline \multirow{2}{*}{ Method } & \multicolumn{5}{|c|}{$a / h$} \\
\cline { 2 - 6 } & 5 & 10 & 20 & 50 & 100 \\
\hline \hline CPT (Khdeir and Librescu, 1988) & 36.160 & 36.160 & 36.160 & 36.160 & 36.160 \\
\hline FSDT (Khdeir and Librescu, 1988) & 11.575 & 23.453 & 31.707 & 35.356 & 35.955 \\
\hline TSDT (Khdeir and Librescu, 1988) & 11.997 & 23.340 & 31.660 & 35.347 & 35.953 \\
\hline IGA FSDT quadratic (Thai et al., 2012) & - & 23.6599 & 31.8288 & 35.3945 & 36.0130 \\
\hline IGA FSDT cubic (Thai et al., 2012) & - & 23.6594 & 31.8267 & 35.3813 & 35.9617 \\
\hline IGA FSDT quartic (Thai et al., 2012) & - & 23.6594 & 31.8267 & 35.3813 & 35.9616 \\
\hline IGA TSDT quadratic & 11.8270 & 23.1558 & 31.5738 & 35.3480 & 35.9807 \\
\hline IGA TSDT cubic & 11.8135 & 23.1386 & 31.5541 & 35.3245 & 35.9474 \\
\hline IGA TSDT quartic & 11.8134 & 23.1385 & 31.5540 & 35.3243 & 35.9468 \\
\hline
\end{tabular}

\subsection{Square plate under biaxial compression}

The last numerical example in this paper considers a symmetric $\left[0^{\circ} / 90^{\circ} / 0^{\circ}\right]$ three-layer simply supported plate with the same material characteristics as in the previous example, subjected to the biaxial buckling load (Fig. 6). the dimensionless buckling factor is defined in the same way as in the uniaxial compression example. The results presented in Table 7 show the dimensionless buckling factor for different length-to-thickness ratios. The results obtained by the proposed method are in good agreement with CPT, FSDT and TSDT solutions by Khdeir and Librescu (1988).

\section{Conclusions}

The current investigation presents the isogeometric free vibration and buckling analysis of a laminated plate based on the TSDT theory of Reddy. TSDT is chosen in order to avoid the usage of shear correction factors. By using NURBS basis functions, the C1 continuity needed for the implementation of TSDT in FEM is easily achieved. It is relatively easy and straightforward to change the order of NURBS basis functions, so quadratic, cubic, quartic or higher order TSDT elements are easily formulated. The presented results are very accurate and close to analytical 
Table 7. Normalized critical buckling load of the simply supported $\left[0^{\circ} / 90^{\circ} / 0^{\circ}\right]$ cross-ply plate under biaxial compression

\begin{tabular}{|l|c|c|c|c|c|}
\hline \multirow{2}{*}{ Method } & \multicolumn{4}{|c|}{$a / h$} & \\
\cline { 2 - 6 } & 5 & 10 & 20 & 50 & 100 \\
\hline \hline CPT (Khdeir and Librescu, 1988) & 14.704 & 14.704 & 14.704 & 14.704 & 14.704 \\
\hline FSDT (Khdeir and Librescu, 1988) & 1.427 & 5.492 & 10.202 & 12.192 & 13.146 \\
\hline TSDT (Khdeir and Librescu, 1988) & 1.465 & 5.526 & 10.259 & 12.226 & 13.285 \\
\hline IGA TSDT quadratic & 1.4262 & 5.2755 & 9.7590 & 11.9065 & 12.9697 \\
\hline IGA TSDT cubic & 1.4198 & 5.2670 & 9.7455 & 11.8873 & 12.9437 \\
\hline IGA TSDT quartic & 1.4198 & 5.2670 & 9.7453 & 11.8868 & 12.9428 \\
\hline
\end{tabular}

TSDT solutions. It can be seen that for the free vibration and buckling analyses, cubic and quartic elements give very similar results, while quadratic elements provide slightly less accurate results. In our opinion, one can use cubic TSDT elements in order to obtain satisfactory results in the least computationally expensive way.

Isogeometric analysis is not confined only to NURBS basis functions. Since NURBS basis functions have several disadvantages from the point of view of analysis, such as the inability of local refinement, a considerable effort is invested in the research of T-splines (Bazilevs et al., 2010), locally refined (LR) B-splines (Johannessen et al., 2014), PHT splines (Nguyen-Thanh et al., 2011), hierarchical refinement of NURBS (Schillinger et al., 2012) and other technologies that are capable of local refinement in the context of isogeometric analysis.

In this paper, the proposed method is used on simple geometries, but it is possible to deal with more complex geometries by using T-spline technique or the bending strip method proposed by Kindl et al. (2010).

Acknowledgment

This work has been supported by the Ministry of Science and Technological Development of Republic of Serbia through Technological Development Project No. 35035.

\section{References}

1. Bazilevs Y., Calo V.M.,Cottrell J.A., Evans J.A., Hughes T.J.R., Lipton S., Scott M.A., Sederberg T.W., Isogeometric analysis using T-splines, Computer Methods in Applied Mechanics and Engineering, 199, 229-263

2. Benson D.J., Bazilevs Y., Hsu M.C., Hughes T.J.R., 2011, Isogeometric shell analysis: the Reissner-Mindlin shell, Computer Methods in Applied Mechanics and Engineering, 199, 5/8, $276-289$

3. Casanova C., Gallego A., 2013, NURBS-based analysis of higher-order composite shells, Composite Structures, 104, 125-133

4. Cottrell J.A., Hughes T.J.R., Bazilevs Y., 2009, Isogeometric Analysis: Toward Integration of $C A D$ and FEA, John Wiley \& Sons, Chichester

5. Echter R., Oesterle B., Bischoff M., 2013, A hierarchic family of isogeometric shell finite elements, Computer Methods in Applied Mechanics and Engineering, 254, 170-180

6. Hughes T.J.R., Cottrell J.A., Bazilevs Y., 2005, Isogeometric analysis: CAD, finite elements, NURBS, exact geometry and mesh refinement, Computer Methods in Applied Mechanics and Engineering, 194, 39/41, 4135-4195

7. Johannessen K.A., Kvamsdal T., Dokken T., 2014, Isogeometric analysis using LR B-splines, Computer Methods in Applied Mechanics and Engineering, 269, 471-514 
8. Jones R.M., 1999, Mechanics of Composite Materials, 2nd ed., Taylor \& Francis, Philadelphia

9. Khdeir A.A, Librescu L., 1988, Analysis of symmetric cross-ply laminated elastic plates using a higher-order theory: Part II - Buckling and free vibration, Composite Structures, 9, 4, 259-277

10. Kiendl J., Bazilevs Y., Hsu M.-C., Wchner R., Bletzinger K.-U., 2010, The bending strip method for isogeometric analysis of Kirchhoff-Love shell structures comprised of multiple patches, Computer Methods in Applied Mechanics and Engineering, 199, 37/40, 2403-2416

11. Kiendl J., Bletzinger K.-U., Linhard J., Wchner R., 2009, Isogeometric shell analysis with Kirchhoff-Love elements, Computer Methods in Applied Mechanics and Engineering, 198, 49/52, 3902-3914

12. Liew K.M., Huang Y.Q., Reddy J.N., 2003, Vibration analysis of symmetrically laminated plates based on FSDT using the moving least squares differential quadrature method, Computer Methods in Applied Mechanics and Engineering, 192, 2203-2222

13. Nguyen-Thanh N., Kiendl J., Nguyen-Xuan H., Wchner R., Bletzinger K.U., Bazilevs Y., RABCZUK T., 2011, Rotation free isogeometric thin shell analysis using PHT-splines, Computer Methods in Applied Mechanics and Engineering, 200, 47/48, 3410-3424

14. Nguren-VAn H., Mai-Duy N., Tran-Cong T., 2008, Free vibration analysis of laminated plate/shell structures based on FSDT with a stabilized nodal-integrated quadrilateral element, Journal of Sound and Vibration, 313, 1/2, 205-223

15. Nosier A., Kapania R.K., Reddy J.N., 1993, Free vibration analysis of laminated plates using a layerwise theory, AIAA Journal, 31, 12, 2335-2346

16. Piegl L., Tiller W., 1997, The NURBS Book, Springer-Verlag New York, New York

17. RedDy J.N., 1984, A simple higher-order theory for laminated composite plates, Journal of Applied Mechanics, 51, 4, 745-752

18. RedDy J.N., 2004, Mechanics of Laminated Composite Plates and Shells Theory and Anlysis, 2nd ed., CRC Press, Boca Raton

19. Rogers D., 2001, An Introduction to NURBS With Historical Perspective, Morgan Kaufmann Publishers, San Francisco

20. Schillinger D., Dedè L., Scott M.A., Evans J.A., Borden M.J., Rank E., Hughes T.J.R., 2012, An isogeometric design-through-analysis methodology based on adaptive hierarchical refinement of NURBS, immersed boundary methods, and T-spline CAD surfaces, Computer Methods in Applied Mechanics and Engineering, 249/252, 116-150

21. Shojaee S., Valizadeh N., Izadpanah E., Bui T., Vu T.-V., 2012, Free vibration and buckling analysis of laminated composite plates using the NURBS-based isogeometric finite element method, Composite Structures, 94, 5, 1677-1693

22. Thai C., Ferreira A.J.M., Carrera E., Nguyen-Xuan H., 2013, Isogeometric analysis of laminated composite and sandwich plates using a layerwise deformation theory, Composite Structures, 104, 196-214

23. Thai C.H., Nguyen-Xuan H., Nguyen-Xuan N., Le T-H., Nguyen-Thoi T., Rabczuk T., 2012, Static, free vibration, and buckling analysis of laminates composite Reissner-Mindlin plates using NURBS-based isogeometric approach, International Journal for Numerical Methods in Engineering, 91, 6, 571-603 